\title{
AVALIAÇÃO DA RELAÇÃO DO DESVIO DE SEPTO COM A OBSTRUÇÃO DO DUCTO NASOLACRIMAL PELA TOMOGRAFIA COMPUTADORIZADA HELICOIDAL
}

\author{
Daniela Signorelli Nunes SILVA ${ }^{1}$ \\ Rodrigo de Andrade RUFINO² \\ Maria Jose APS TUCUNDUVA ${ }^{3}$
}

\section{RESUMO}

O ducto nasolacrimal é um canal que tem início na porção medial anterior do assoalho da órbita e que deságua na cavidade nasal, no meato inferior. Por meio desse ducto a lágrima é recolhida e drenada da órbita para a cavidade nasal. A obstrução do ducto nasolacrimal pode apresentar diferentes etiologias, podendo ser doenças ou alterações anatômicas. Alterações no padrão de normalidade da relação entre o ducto nasolacrimal e o septo podem favorecer a obstrução do ducto. Com isso, faz-se necessário avaliar a distância normal entre o septo nasal e o ducto nasolacrimal, por meio de tomografia computadorizada de modo a conhecermos o padrão de distância comumente encontrado e analisar alterações desse padrão e a contribuição do desvio que possam levar à perda da saúde nessa região.

Neste trabalho, foi utilizada uma amostra contendo 51 exames de tomografia computadorizada helicoidal da face, de ambos os gêneros, sendo 22 homens (43,13\%), faixa etária entre 18 e 96 anos, e 29 mulheres (56,87\%), faixa etária entre 20 e 73 anos. A análise das imagens foi feita em duas alturas, sendo a primeira ao nível da órbita e a segunda ao nível da raiz da concha nasal inferior e, então, foram mensurados, pelo programa Microdicon ${ }^{\circledR}$, o comprimento e a largura do canal nasolacrimal e sua distância ao septo nasal. Em seguida, sofreram tratamento estatístico com técnicas não paramétricas.

Devido ao desvio de septo das amostras, as distâncias entre o ducto nasolacrimal e o septo nas duas alturas apresentaram discrepância entre os valores. $E$ a largura na altura da concha nasal inferior também apresentou discrepância, entretanto o valor de $p$ foi 0,51, sugerindo que o resultado seja consequência do acaso. Com isso, não obtivemos resultados que possam correlacionar as dimensões do canal nasolacrimal com a direção do desvio do septo e a obstrução do ducto.

PALAVRAS-CHAVE: DUCTO NASOLACRIMAL - SEPTO NASAL - TOMOGRAFIA COMPUTADORIZADA ESPIRAL.

1 Graduanda do curso de medicina na Universidade Municipal de São Caetano do Sul. Ingresso em 2014. Conclusão em 2019.

2 Graduado em biomedicina pela UNICID, mestrado em ciências morfofuncionais pelo instituto de ciências biomédicas da USP, atualmente professor do curso de biomedicina da UNICID.

3 Graduada em odontologia pela UNICID, doutorado em diagnóstico bucal pela faculdade de odontologia da USP, atualmente professora dos cursos de medicina e biomedicina da UNICID e curso de medicina da USCS. 


\section{EVALUATION OF THE RELATION OF THE SEPTUM DEVIATION WITH THE OBSTRUCTION OF THE LACRIMONASAL DUCT BY HELICAL COMPUTED TOMOGRAPHY}

\section{ABSTRACT}

The nasolacrimal duct is a canal that begins in the anterior medial portion of the floor of the orbit and drains into the nasal cavity in the inferior meatus. Through this duct the tear is collected and drained from the orbit into the nasal cavity. The obstruction of the nasolacrimal duct may present different etiologies, including diseases and anatomical alterations. Changes in the normality of the relationship between the nasolacrimal duct and the septum may favor the duct obstruction. Thus, it is necessary to evaluate the normal distance between the nasal septum and the nasolacrimal duct by means of computerized tomography in order to know the distance pattern commonly found and to analyze changes in this pattern and the contribution of the deviation that could lead to health loss in this region.

In this study, a sample containing 51 helicoidal computerized tomography (CT) examinations of both genders was used: 22 men (43.13\%), aged between 18 and 96 years old, and 29 women (56.87\%), aged between 20 and 73 years old. The images were analyzed at two different heights, the first at orbit level and the second at the root level of the inferior turbinate, and then, measured by the Microdicon ${ }^{\oplus}$ program, the length and width of the nasolacrimal canal and its distance to the nasal septum. Afterwards, they underwent statistical treatment with non-parametric techniques.

Due to the septal deviation of the samples, the distances between the nasolacrimal duct and the septum at both heights presented a discrepancy between the values. And the width at the height of the inferior turbinate also presented a discrepancy, however the $p$ value was 0.51 , suggesting that the result is a consequence of chance. With this, we did not obtain results that could correlate the dimensions of the lacrimonasal canal with the direction of the septal deviation and the duct obstruction.

KEY WORDS: NASOLACRIMAL DUCT - NASAL SEPTUM - TOMOGRAPHY, SPIRAL COMPUTED.

\section{INTRODUÇÃO}

A cavidade nasal, segundo Moore et al.' (2014), é dividida em cavidades direita e esquerda, pelo septo nasal. Este é formado por uma parte óssea e uma parte cartilagínea móvel flexível. É comum ocorrer o desvio do septo nasal para um dos lados da cavidade nasal e, quanto mais acentuado, maior a possibilidade de obstrução nasal.

Os seios paranasais, segundo Junqueira e Carneiro² (2013), são formados por epitélio do tipo respiratório e possuem células caliciformes, formadoras de muco. O muco é drenado para a cavidade nasal com o auxílio das células ciliadas.

O meato nasal inferior se liga, em sua parte anterior, ao ducto nasolacrimal, este compõe o aparelho lacrimal que é formado por: glândula lacrimal, dúctulos excretores da glândula lacrimal, canalículos lacrimais e ducto nasolacrimal.

A obstrução do ducto nasolacrimal pode apresentar diferentes etiologias e, segundo Navarro et al. ${ }^{3}$, em 2013, pode ocorrer em qualquer ponto de sua rota. Algumas doenças, como a sarcoidose, descrita por 
ISSN 1983-5183

Sardinha et al. ${ }^{4}$ (2004), podem acometer o ducto nasolacrimal, causando a obstrução do canal e favorecendo a dacriocistite, inflamação do saco lacrimal, podendo ser primariamente adquirida ou secundária à própria obstrução do ducto nasolacrimal ou da cavidade nasal. Outra afecção que pode estar associada à obstrução do ducto nasolacrimal é a mucocele dos seios paranasais e do ducto nasolacrimal, descrita por Carvalho et al..$^{5}$ (2013), e Dores et al. ${ }^{6}$ (2012); essa pode invadir as estruturas orbitais e provocar a obstrução do ducto; entretanto, o acometimento do sistema lacrimal é uma manifestação atípica. Alterações na prevalência das células, como as da crista do nariz do etmoide, descritas por Marques et al. ${ }^{7}$ (2011), por estarem relacionadas anatomicamente com o ducto nasolacrimal, assim como algumas variações na morfologia do canal podem favorecer a obstrução, interrompendo o fluxo do líquido lacrimonasal. Além disso, Lourenço ${ }^{8}(2006)$ associou alterações anatômicas e infecções à obstrução nasal, o que também pode favorecer a obstrução do ducto lacrimonasal.

Para avaliação das estruturas da cavidade nasal e dos seios paranasais, a fim de identificar as possíveis causas da obstrução do ducto nasolacrimal, segundo Bolzan e Tucunduva ${ }^{9}$ (2012), é indicado o uso de tomografia computadorizada (TC), pois possibilita análise detalhada devido à redução na sobreposição de imagens, relacionadas as radiografias. Em concordância com a escolha de método, Bulbul et al..$^{10}$ em 2016 avaliaram a diferença morfológica da obstrução do ducto lacrimonasal por TC.

Por meio de 51 exames de tomografia computadorizada helicoidal, foram observados cortes axiais em duas alturas, junto à órbita e junto à concha nasal inferior, identificando o ducto nasolacrimal, mensurando seu comprimento e largura, possibilitando, assim, a mensuração da distância até o septo nasal e posterior tratamento estatístico.

O presente estudo tem como objetivo avaliar, por meio de tomografia computadorizada helicoidal, a distância do ducto nasolacrimal e o septo e, comparando ao padrão de normalidade, detectar alterações que possam contribuir com a obstrução do ducto nasolacrimal.

\section{OBJETIVO}

Estabelecer o padrão de normalidade do trajeto do ducto nasolacrimal. Isso permite, quando diante de uma alteração, sua detecção. A mensuração da distância do ducto ao septo permite o estabelecimento desse parâmetro.

O presente estudo tem como objetivo avaliar, por meio de tomografia computadorizada helicoidal, a distância do ducto nasolacrimal e o septo e, comparando ao padrão de normalidade, detectar alterações que possam contribuir com a obstrução do ducto nasolacrimal.

\section{MATERIAL E MÉTODOS}

As imagens que foram analisadas são provenientes do banco de dados de imagens de tomografia computadorizada de seios da face que foi disponibilizado para uso no presente trabalho, mediante autorização do guardião do banco de imagens e do CEP da Universidade Municipal de São Caetano do Sul - USCS (CAAE - 56126316.2.0000.5510).

As imagens supracitadas foram obtidas em tomógrafo computadorizado helicoidal (Somaton AR Star/Siemens), com regime de trabalho de $83 \mathrm{~mA}$ e $110 \mathrm{kV}$. O protocolo utilizado foi caracterizado por cortes axiais de $2 \mathrm{~mm}$ de espessura e $5 \mathrm{~mm}$ de incremento e cortes coronais de $3 \mathrm{~mm}$ de espessura e $3 \mathrm{~mm}$ de incremento. Os cortes foram adquiridos com o paciente em posição de decúbito dorsal, sem a introdução 
Silva DSN, Rufino RA, Tucunduva MJAPS Avaliação da relação do desvio de septo com a obstrução do ducto nasolacrimal pela tomografia computadorizada helicoidal. Rev. Odontol. Univ. Cid. São Paulo 2018 jan-mar; 30(1): 47-53

ISSN 1983-5183

de meio de contraste ou uso de descongestionante. Os cortes coronais foram iniciados na compacta externa do osso frontal, seguindo perpendicularmente ao palato ósseo, com limite posterior na parede posterior do osso esfenoide; e os cortes axiais tiveram como limite os tecidos moles.

A amostra, no presente estudo, contou com indivíduos de ambos os gêneros, sendo 22 homens e 29 mulheres, com idades entre 18 e 96 anos, sendo a média aproximada das idades 42 anos. As imagens foram analisadas a partir de 51 exames de tomografia computadorizada da face.

Foram considerados critérios de exclusão exames de pacientes que reportaram cirurgia prévia e pacientes com idade inferior a 18 anos de vida cronológica, devido a alterações no crescimento das estruturas faciais. As imagens analisadas constaram de cortes axiais em duas alturas, sendo a primeira ao nível da órbita e a segunda ao nível da raiz da concha nasal inferior. Nesses cortes foram mensurados o comprimento e a largura do canal lacrimonasal e sua distância ao septo nasal. As imagens foram observadas em local de baixa luminescência, na tela do monitor, em um computador dos laboratórios de informática da USCS, utilizando-se o programa Medwork 7.65 para visualização das imagens. A mensuração foi realizada pelo programa MicroDicon ${ }^{\circledR}$. Os dados obtidos foram tabulados em planilha do Excel e sofreram tratamento estatístico com técnicas não paramétricas.

\section{RESULTADOS}

Foram analisados 51 exames de tomografia computadorizada da face, sendo 22 masculinos (43,13\%), faixa etária entre 18 e 96 anos, e 29 femininos (56,87\%), faixa etária entre 20 e 73 anos. Sendo assim, houve um predomínio do sexo feminino nas amostras, impossibilitando relacionar o sexo com os resultados.

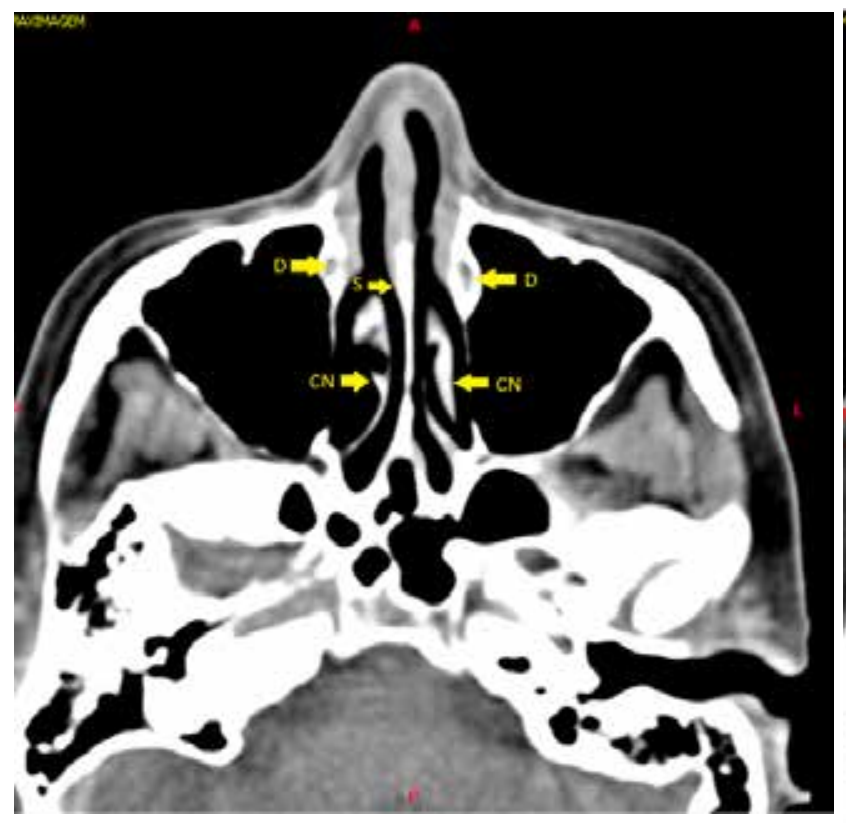

Figura 1: Tomografia computadorizada de face, corte axial, altura da concha nasal inferior. D: Ducto nasolacrimal; S: Septo; CN: Concha nasal inferior

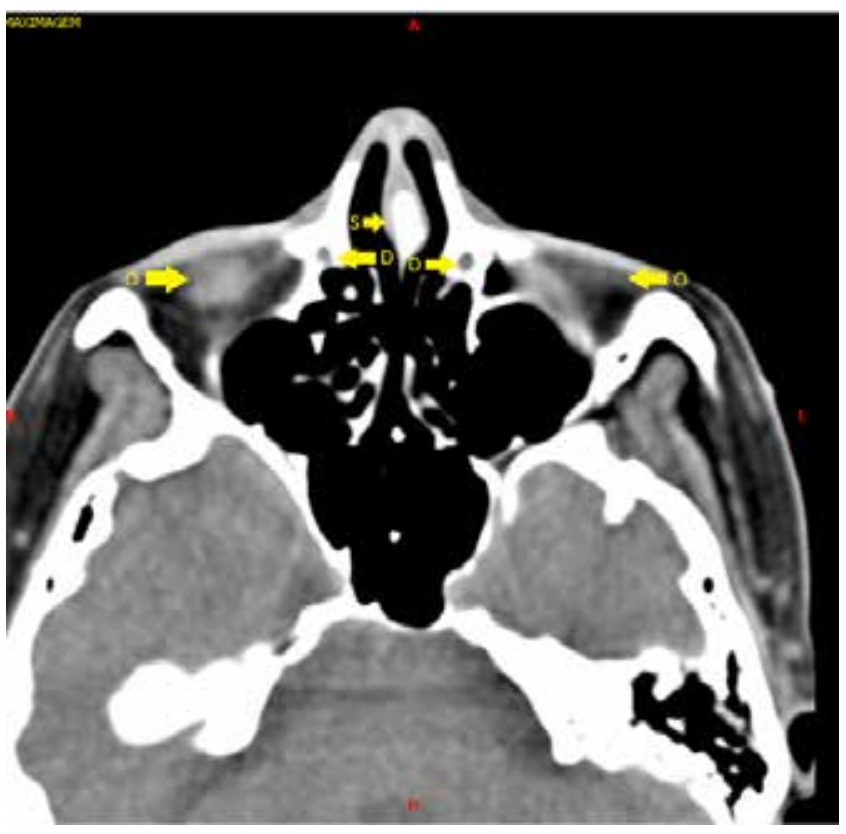

Figura 2: Tomografia computadorizada de face, corte axial, altura da órbita. D: Ducto nasolacrimal; S: Septo; O: Órbita

Após a mensuração, realizada pelo programa MicroDicon ${ }^{\circledast}$, foram obtidos dados quantitativos relacionados ao canal nasolacrimal, comprimento e largura, nas alturas da concha nasal inferior e da órbita, 
Silva DSN, Rufino RA, Tucunduva MJAPS Avaliação da relação do desvio de septo com a obstrução do ducto nasolacrimal pela tomografia computadorizada helicoidal. Rev. Odontol. Univ. Cid. São Paulo 2018 jan-mar; 30(1): 47-53

ISSN 1983-5183

e a distância entre o canal nasolacrimal e o septo, nas hemifaces direita e esquerda.

Na altura da concha nasal inferior, na hemiface direita, obtivemos um comprimento médio de 19,57mm; a largura média de 12,91mm; e a distância média do canal nasolacrimal ao septo de 38,23mm. Na hemiface esquerda, o comprimento médio de 19,07mm; a largura média de 12,79mm; e a distância média do canal nasolacrimal ao septo de $41,68 \mathrm{~mm}$.

Na altura da órbita, na hemiface direita, obtivemos o comprimento médio de 18,37mm; a largura média de 15,14mm; e a distância média do canal nasolacrimal ao septo de 39,33mm. Na hemiface esquerda, o comprimento médio de 18,06mm; a largura média de 14,81mm; e a distância média do canal nasolacrimal ao septo de $42,56 \mathrm{~mm}$.

Para avaliação foram utilizadas técnicas de estatística não paramétrica, ou seja, não possui padrão estabelecido estre os valores. Comparando-se as duas variáveis, os lados direito e esquerdo, obtivemos os seguintes resultados:

\section{Comparação Direito e Esquerdo:}
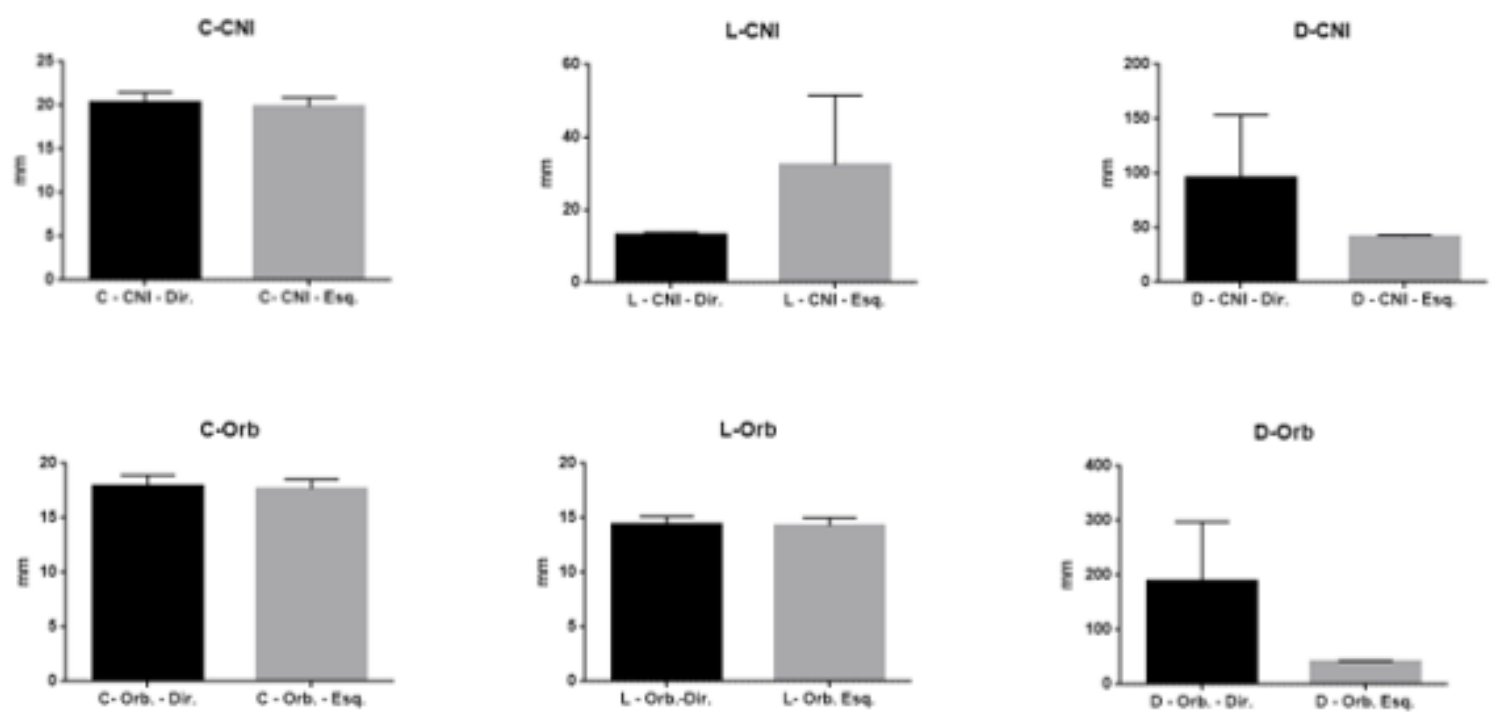

Legenda: C - comprimento; L - largura; D - distância; CNI - concha nasal inferior; Orb - órbita

Os resultados mais discrepantes foram entre as distâncias nas duas alturas, justificadas pelo desvio de septo das amostras, e na largura na altura da concha nasal inferior. Entretanto, um dos parâmetros estatísticos é o valor de $p$ (probabilidade de significância) que só é significativo se $\leq 0,05$, o que demonstraria que o resultado não é consequência do acaso e sim de um fator causal, e, nesse caso, foi 0,51.

Além disso, a distância é maior para o lado direito, portanto, há maior desvio de septos para a esquerda, e a largura é menor no lado direito na altura da concha nasal inferior, não havendo relação clara entre esses resultados. 
ISSN 1983-5183

\section{DISCUSSÃO}

A obstrução do ducto nasolacrimal pode apresentar diferentes etiologias. Segundo Sardinha et al. ${ }^{4}$ (2004), a glândula lacrimal está entre as principais estruturas acometidas pela sarcoidose. Marques et al. ${ }^{7}$ (2011) descreveram o estreitamento do óstio do ducto lacrimonasal pelas células acessórias. Já Lourenço ${ }^{8}$ (2006) elucidou que a obstrução nasal pode provocar alterações anatômicas e maior suscetibilidade a infecções, assim como as mucoceles dos seios paranasais descritas por Dores et al. ${ }^{6}$ (2012). A partir dos resultados desse estudo, conclui-se que a obstrução do ducto nasolacrimal não se relaciona com o desvio do septo; sendo assim, as prováveis causas de obstrução seriam doenças que acometem o aparelho lacrimal, causando estreitamento do canal, como descrito por Sardinha et al. ${ }^{4}$ (2004) e Marques et al. ${ }^{7}$ (2011), ou alterações anatômicas e infecções como descrito por Lourenço ${ }^{8}$ (2006) e Dores et al. ${ }^{6}$ (2012).

Foi possível identificar que há maior desvio de septos para a esquerda e a largura do ducto nasolacrimal é menor do lado direito, na altura da concha nasal inferior. Isso demonstra que o desvio do septo não está na direção do estreitamento do canal, o que contribui para a afirmação de que a obstrução do ducto nasolacrimal não se relaciona com o desvio do septo.

\section{CONCLUSÃO}

Conclui-se que a obstrução do ducto nasolacrimal pode apresentar diferentes etiologias, entretanto, não se relaciona com o desvio do septo. Alterações no próprio ducto podem levar a obstrução; com isso, é interessante, em uma pesquisa futura, analisar estatisticamente as dimensões do próprio ducto nas diferentes alturas, determinando possíveis alterações. 


\section{REFERÊNCIAS}

1. MOORE KL, Dalley AF, Agur AMR. Anatomia orientada para a clínica. 7. ed. Rio de Janeiro: Guanabara Koogan; 2014.

2. JUNQUEIRA LC, Carneiro J. Histologia básica. 12. ed. Rio de Janeiro: Guanabara Koogan; 2013.

3. NAVARRO Pde L, Machado Junior AJ, Crespo AN. Evaluation of the lacrimal recess of the maxillary sinus: an anatomical study. Braz J Otorhinolaryngol 2013 Jan-Feb;79(1):35-8.

4. SARDINHA M, Nunes T, Santo R, Matayoshi S. Dacriocistite crônica secundária a sarcoidose: relato de caso. Arq Bras Oftalmol 2004 out;67(5):827-30.

5. CARVALHO BV, Lopes ICC, Corrêa JB, Ramos LFM, Motta EGPC, Diniz RLFC. Apresentações típicas e atípicas de mucocele dos seios paranasais na tomografia computadorizada. Radiol Bras 2013 dez;46(6):372-5.

6. DORES LA, Marques MC, Agostinho S, Simão MA, Santos A, Dias Ó, et al. Mucocelos dos seios perinasais: Considerações em cinco casos clínicos. Rev Portuguesa Otorrinolaringologia Cirurgia Cérvico-Facial 2012 50(2):6.

7. MARQUES MC, Simão MA, Santos A, Macor C, Dias Ó, Andrea M. Análise da anatomia do recesso frontal em tomografia computorizada: Estudo de 50 doentes. Rev Portuguesa Otorrinolaringologia Cirurgia Cérvico-Facial 2011 49(1):6.

8. LOURENÇO EA. Obstrução nasal: valorize este sintoma e conheça a orientação terapêutica. Perspectivas Médicas 2006 jan-dez;17(1):42-4.

9. BOLZAN JD, Tucunduva MJAPS. Estudo radiográfico da cavidade nasal e dos seios paranasais e suas variações. Scien Health 2012 jan-abr ;3(1):23-31.

10. BULBUL E, Yazici A, Yanik B, Yazici H, Demirpolat G. Morphometric evaluation of bony nasolacrimal canal in a caucasian population with primary acquired nasolacrimal duct obstruction: a multidetector computed tomography study. Korean J Radiol 2016 Mar-Apr;17(2):271-6.

RECEBIDO EM

ACEITO EM 23/10/2017 